Check for updates

Cite this: Chem. Sci., 2019, 10, 440

๑ All publication charges for this article have been paid for by the Royal Society of Chemistry

Received 10th September 2018 Accepted 10th October 2018

DOI: $10.1039 / c 8 s c 04006 k$

rsc.li/chemical-science

\section{A versatile synthetic strategy for macromolecular cages: intramolecular consecutive cyclization of star-shaped polymers $\uparrow$}

\author{
Yoshinobu Mato, ${ }^{a}$ Kohei Honda, ${ }^{a}$ Kenji Tajima, ${ }^{b}$ Takuya Yamamoto, (D) ${ }^{\text {b }}$ \\ Takuya Isono (D) ${ }^{* b}$ and Toshifumi Satoh (D) *b
}

Cage-shaped polymers, or "macromolecular cages", are of great interest as the macromolecular analogues of molecular cages because of their various potential applications in supramolecular chemistry and materials science. However, the systematic synthesis of macromolecular cages remains a great challenge. Herein, we describe a robust and versatile synthetic strategy for macromolecular cages with defined arm numbers and sizes based on the intramolecular consecutive cyclization of highly reactive norbornene groups attached to each end of the arms of a star-shaped polymer precursor. The cyclizations of three-, four-, six-, and eight-armed star-shaped poly( $\varepsilon$-caprolactone)s (PCLs) bearing a norbornenyl group at each arm terminus were effected with Grubbs' third generation catalyst at high dilution. ${ }^{1} \mathrm{H}$ NMR, SEC, and MALDI-TOF MS analyses revealed that the reaction proceeded to produce the desired macromolecular cages with sufficient purity. The molecular sizes of the macromolecular cages were controlled by simply changing the molecular weight of the star-shaped polymer precursors. Systematic investigation of the structure-property relationships confirmed that the macromolecular cages adopt a much more compact conformation, in both the solution and bulk states, as compared to their linear and star-shaped counterparts. This synthetic approach marks a significant advance in the synthesis of complex macromolecular architectures and provides a platform for novel applications using cage-shaped molecules with polymer frameworks.

\section{Introduction}

Molecular cages have drawn attention as intriguing chemical research subjects because of their structural uniqueness, featuring three-dimensional cavities inside their molecular frameworks. ${ }^{1-4}$ Owing to such distinctive architectures, cage molecules have been utilized for a diverse range of applications, such as nanocapsules for relatively large molecules, ${ }^{5,6}$ templates for the size-controlled synthesis of metal nanoparticles, ${ }^{7}$ and nanoflasks for catalytic reactions. ${ }^{8,9}$ Cage molecules can be constructed by either the formation of covalent bonds or selfassembly through non-covalent bonding interactions. A number of small organic cages such as cryptands have been synthesized mainly by the former approach. ${ }^{10,11}$ On the other hand, the latter approach has recently proven to be a powerful means to construct giant cages such as DNA and protein cages

${ }^{a}$ Graduate School of Chemical Sciences and Engineering, Hokkaido University, Sapporo 060-8628, Japan

${ }^{b}$ Division of Applied Chemistry, Faculty of Engineering, Hokkaido University, Sapporo 060-8628, Japan. E-mail: isono.t@eng.hokudai.ac.jp; satoh@eng.hokudai.ac.jp

$\dagger$ Electronic supplementary information (ESI) available: Experimental procedures and additional data $\left({ }^{1} \mathrm{H}\right.$ NMR, MALDI-TOF MS, SEC, DSC, SAXS, and WAXD). See DOI: $10.1039 / \mathrm{c} 8 \mathrm{sc} 04006 \mathrm{k}$ as well as 3D metallocages with a molecular size range of 1-50 nm. ${ }^{12-15}$ Remarkably, the robustness of the self-assembly approach has enabled the creation of novel cage molecules possessing various sizes, topologies, and functionalities.,16 However, the non-covalent bonding interactions are highly sensitive to external conditions, so that the self-assembled cages are relatively fragile upon exposure to chemical/physical stimuli including $\mathrm{pH}$, temperature, and solvent polarity. To further extend the possible applications of cage molecules, a novel covalent-bond-forming strategy for the systematic synthesis of cage molecules with tunable functionality and controllable cavity size is highly desired.

To achieve this goal, we envisioned the use of synthetic polymers as building blocks for the cage-shaped framework. A major advantage of a synthetic polymer is that a cage molecule of a targeted size can be readily synthesized by simply tuning its degree of polymerization. Moreover, we anticipated that the choice of comonomers and their sequence would endow the cage molecules with vast functional utility such as molecular recognition ability, external stimuli responsiveness, and the ability to self-assemble into higher-order structures. ${ }^{17-20}$ However, only limited efforts have been made thus far to prepare macromolecular cages, and therefore, a general synthetic strategy remains lacking. To date, macromolecular 

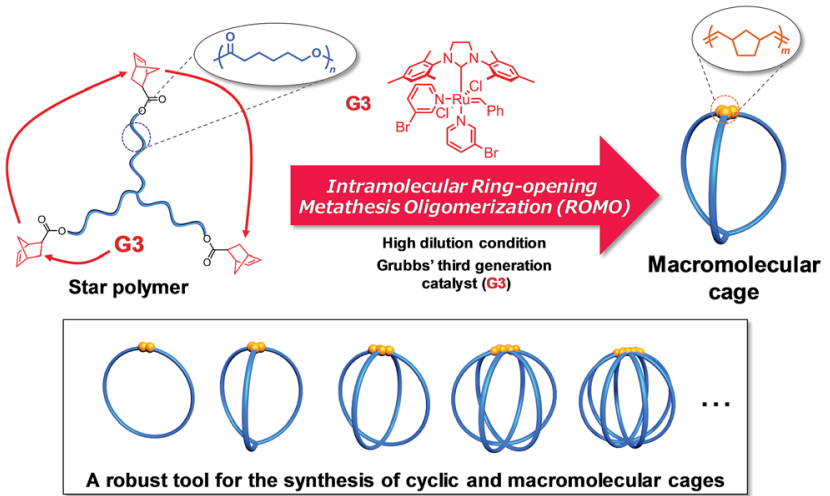

Fig. 1 Schematic illustration of the synthetic strategy for macromolecular cages through intramolecular consecutive ROMO mediated by G3.

cages composed of up to four arms have been reported by the groups of Tezuka and Paik, and more recently, our own. ${ }^{21-24}$ Although each synthesis produced well-defined macromolecular cages, the laborious and multistep natures of these conventional syntheses present a practical limitation to the systematic synthesis of macromolecular cages with arm numbers greater than five. Consequently, the structures and properties of macromolecular cages, especially with respect to the molecular weight and arm number, have never been systematically studied. To achieve the systematic synthesis of macromolecular cages with varying arm numbers, we devised a novel synthetic approach which involves intramolecular cyclization in a chain-reaction manner to construct a multi-ring system. Such chain reactiontype cyclization would be ideal for the step-efficient synthesis of macromolecular cages with various arm numbers. As shown in Fig. 1, the intramolecular consecutive cyclization of star-shaped polymers bearing a polymerizable group at each chain end would enable the systematic synthesis of macromolecular cages.

To selectively produce the desired macromolecular cage, it is essential to suppress possible intermolecular propagation reactions by applying highly dilute conditions. Considering the extraordinarily high reactivity of ring-opening metathesis polymerization (ROMP) coupled with the Grubbs $3^{\text {rd }}$ generation catalyst (G3), ${ }^{25-27}$ the exo-norbornene group was selected as the polymerizable end group. Meanwhile, we employed $\operatorname{poly}(\varepsilon$-caprolactone) (PCL) as the macromolecular framework of the cage due to its ease of preparation with sufficient chainend fidelity and narrow dispersity $(\theta,<1.1) .{ }^{28}$ We herein demonstrate, for the first time, the systematic synthesis of macromolecular cages with varied arm numbers and molecular weights by the intramolecular ring-opening metathesis oligomerization (ROMO) of exo-norbornene groups attached to each chain end of the star-shaped PCLs. In addition, we demonstrate a comprehensive study of the correlation between the cage-shaped structures and polymer properties by employing a series of cage-shaped PCLs.

\section{Results and discussion}

As a model reaction system, we initially optimized the reaction conditions for the intramolecular consecutive ROMO of an $\alpha, \omega-$ dinorbornenyl end-functionalized linear PCL precursor, Pre $_{\text {ring-a, to afford the corresponding monocyclic polymer ring-a }}$

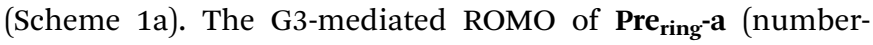
average molecular weight $\left(M_{\mathrm{n}}\right)$ estimated by ${ }^{1} \mathrm{H} \quad \mathrm{NMR}$ $\left(M_{\mathrm{n}, \mathrm{NMR}}\right)=5510, M_{\mathrm{n}}$ estimated by SEC-RI using PSt standards $\left(M_{\mathrm{n}, \mathrm{SEC}}\right)=9790, D=1.05$; see Section S1-3 and Table S1 in the $\mathrm{ESI} \dagger$ for more details) was conducted at high dilution (final polymer precursor concentration $=0.02 \mathrm{mM}$ in $\mathrm{CH}_{2} \mathrm{Cl}_{2}$ ) to preferentially promote the desired intramolecular reaction rather than the undesired intermolecular one. To further minimize intermolecular coupling, the solution of $\mathbf{P r e}_{\text {ring }} \mathbf{- a}$ was added dropwise to the stirred G3 solution in $\mathrm{CH}_{2} \mathrm{Cl}_{2}$ with varying $\left[\right.$ Pre $_{\left.\text {ring }^{-a}\right]_{0} /[\mathrm{G} 3]_{0}}$ ratios of $1 / 1,1 / 2,1 / 4$, and $1 / 6$. Notably, each reaction produced a soluble product, and ${ }^{1} \mathrm{H}$ NMR analysis suggested quantitative consumption of the norbornenyl group (Fig. S1 $\dagger$ ). The molecular weight distribution of the product varies significantly depending upon the $\left[\text { Pre }_{\text {ring }}-\mathbf{a}\right]_{0} /[\mathrm{G} 3]_{0}$ ratio, as shown in Fig. S2.† The size exclusion chromatography (SEC) traces of the products obtained at the $\left[\text { Pre }_{\text {ring }}-\mathbf{a}\right]_{0} /[\mathrm{G} 3]_{0}$ ratios of $1 / 1,1 / 2$, and $1 / 4$ exhibit two elution peak maxima in both higher and lower molecular weight regions with respect to that of Pre $_{\text {ring-a, indicating the formation of intramolecular propaga- }}$ tion and intermolecular cyclization products, respectively. ${ }^{29} \mathrm{On}$ the other hand, the product obtained at the $\left[\text { Pre }_{\text {ring }}-\mathbf{a}\right]_{0} /[\mathrm{G} 3]_{0}$ ratio of $1 / 6$ exhibits a narrowly dispersed unimodal peak in a lower molecular weight region that would be assignable to the intramolecularly constructed product ring-a, as reported previously. ${ }^{30}$ The decrease in the apparent molecular weight after the ROMO with hydrodynamic volume change $(0.75$; calculated from the equation $M_{\mathrm{p}, \mathrm{SEC}\left(\mathrm{Pre}_{\text {ring }}\right)} / M_{\mathrm{p}, \mathrm{SEC}(\mathrm{ring})}$, where $M_{\mathrm{p}, \mathrm{SEC}}$ is the peak-top molecular weight; Fig. S3†) is consistent with the literature range of $0.71-0.83$ for other monocyclic polymers possessing comparable molecular weight. ${ }^{31-33}$ Thus, successful cyclic structure formation is demonstrated for the synthesis of ring-a with suppressed intermolecular propagation. Moreover, the ${ }^{1} \mathrm{H}$ NMR and matrix-assisted laser desorption ionization time-of-flight (MALDI-TOF) mass spectra could be reasonably assigned to the expected chemical structure of ring-a (Fig. S4†). Hence, we found that a [precursor $]_{0} /[\mathrm{G} 3]_{0}$ ratio of $1 / 6$ in combination with a slow addition technique can selectively promote intramolecular ROMO.

Next, we synthesized the three-armed star-shaped PCL bearing a norbornenyl group at each end, Pre $_{3}$-a $\left(M_{\mathrm{n}, \mathrm{NMR}}=\right.$ $6000, M_{\mathrm{n}, \mathrm{SEC}}=9810, D=1.05$ ), which is subject to the envisioned intramolecular ROMO to give the corresponding threearmed macromolecular cage, cage ${ }_{3}$-a (Scheme 1b). The preparation of $\mathbf{P r e}_{3}$-a is successfully achieved in two steps: (i) diphenyl phosphate-catalyzed ring-opening polymerization of $\varepsilon$-caprolactone $(\varepsilon-\mathrm{CL})$ using a commercially available triol initiator with the $[\varepsilon-\mathrm{CL}]_{0} /[\text { initiator }]_{0}$ ratio of $50 / 1$ and (ii) subsequent condensation reaction with excess ( \pm )-exo-5-norbornene carboxylic acid (see Section S1-3†). The ${ }^{1} \mathrm{H}$ NMR spectrum of the product clearly shows the signals attributed to the norbornenyl groups, indicating quantitative introduction of norbornenyl groups to each polymer end (Fig. 2a and S5†). The SEC traces of the obtained product retained monomodal features even after the condensation reaction (Fig. S6†). 
(a)

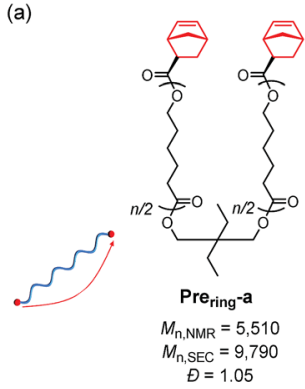

(b)

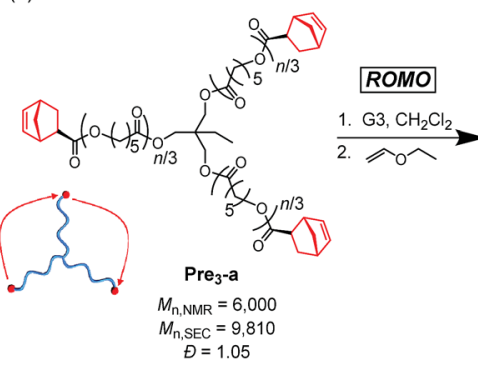

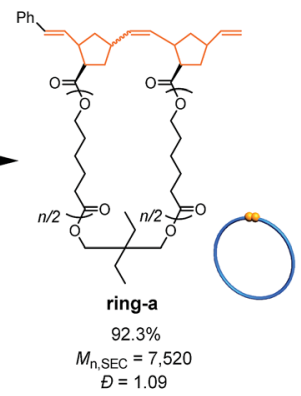

$$
\theta=1.09
$$

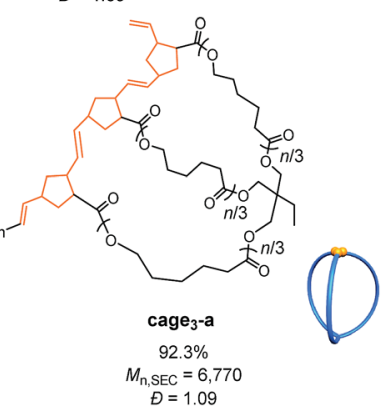

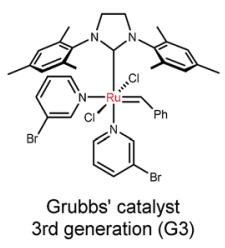

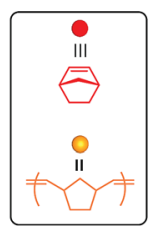

Scheme 1 Synthesis of ring-a (a) and three-armed cage $_{3}-a(b)$ through intramolecular ring-opening metathesis oligomerization (ROMO) of the corresponding PCLs with a reactive norbornenyl group at each chain end.

With the optimized reaction conditions ([precursor $]_{0} /[\mathrm{G} 3]_{0}=$ $1 / 6$; [precursor $]_{0}=0.02 \mathrm{mM}$ ) in hand, the intramolecular consecutive ROMO of Pre $_{3}$-a was carried out to give cage $_{3}$ a (Scheme 1b). In the ${ }^{1} \mathrm{H}$ NMR spectrum of the product, no signals due to the norbornenyl group are detected, whereas signals attributable to the oligonorbornene backbone are
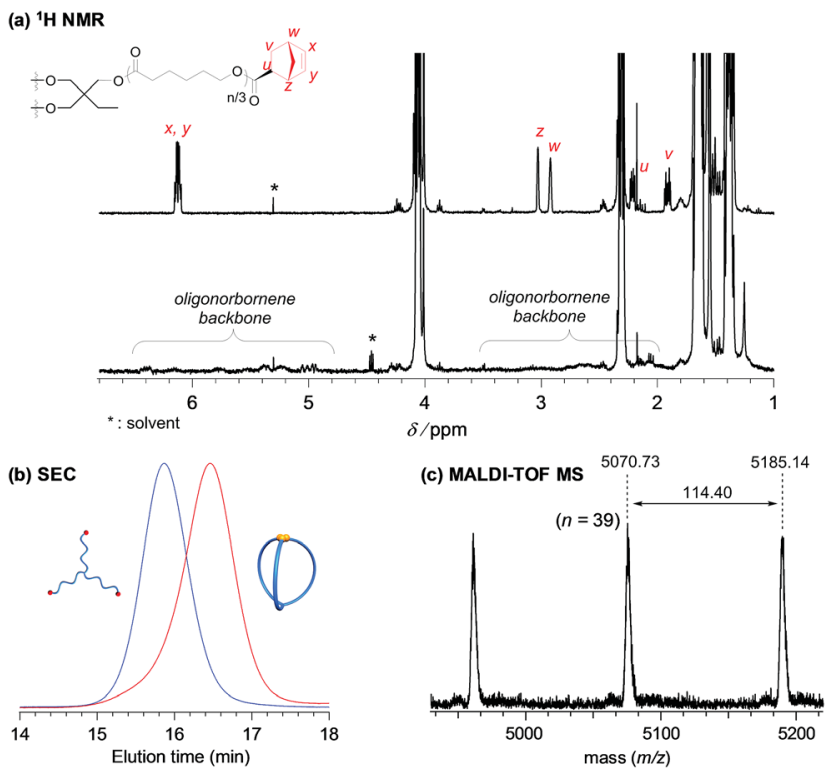

Fig. 2 Structural analysis of the three-armed macromolecular cage (cage $\left.{ }_{3}-a\right)$. (a) ${ }^{1} \mathrm{H}$ NMR spectra of Pre $_{3}-a$ (upper) and cage 3 -a (lower). (b) SEC traces of Pre $3-a\left(M_{n, N M R}=6000, M_{n, S E C}=9810, \emptyset=1.05\right.$; blue line) and cage $_{3}$-a (before purification by preparative SEC; $M_{n, S E C}=$ $6770, \ominus=1.09$; red line) synthesized by intramolecular ROMO. (c) Expanded MALDI-TOF MS spectrum of cage $_{3}$-a ranging from 4500 to $5200 \mathrm{Da}$ observed near 1.05-3.25 and 4.95-6.60 ppm (Fig. 2a). Although the ${ }^{1} \mathrm{H}$ NMR analysis revealed the completion of the reaction, several possible side reactions such as intermolecular polymerization and the multiple addition of G3 were also considered (Fig. S8†). To exclude the possibilities of such side reactions, SEC and MALDI TOF-MS analyses were performed to further verify the detailed structure of the product. The SEC trace of the obtained product is significantly shifted to the lower molecular region $\left(M_{\mathrm{n}, \mathrm{SEC}}=6770, Ð=1.09\right)$ as compared to Pre $_{3}-\mathbf{a}\left(M_{\mathrm{n}, \mathrm{SEC}}=9810\right)$, implying the formation of the desired cage-shaped product with a smaller hydrodynamic volume (Fig. $2 \mathrm{~b}$ and $\mathrm{S} 6 \dagger$ ).

Furthermore, the monomodal elution peak is retained after the reaction, suggesting that the intermolecular reaction is highly suppressed. Although a small higher molecular weight shoulder, perhaps due to the dimer and trimer formed via the intermolecular propagation, was observed in the SEC trace, the purity of the desired product was calculated to be higher than $90 \%$ based on the elution peak area (Table S2 $\dagger$ ). Moreover, the MALDI-TOF mass spectrum shows only one set of peaks with a regular interval of $114.40 \mathrm{Da}$ corresponding to the $\varepsilon$-CL monomer unit, which is a good indication that multiple G3 addition did not occur (Fig. $2 \mathrm{c}$ and $\mathrm{S} 7 \dagger$ ). Specifically, peaks due to possible multiple G3 adducts, such as the tadpole- (for example $[\mathrm{M}+\mathrm{Na}]^{+}=5059.97 \mathrm{Da}, n=38$ ) and star-shaped polymers (for example $[\mathrm{M}+\mathrm{Na}]^{+}=5165.03 \mathrm{Da}, n=38$, Fig. S8 $\dagger$ ), are not detected. In addition, an observed peak at $\mathrm{m} / \mathrm{z}$ 5070.73 Da agrees with the calculated mass for the desired cage $_{3}$-a with a degree of polymerization of $39\left([\mathrm{M}+\mathrm{Na}]^{+}=\right.$ $5070.05 \mathrm{Da}, n=39)$. Overall, these data strongly confirm that the intramolecular consecutive ROMO using G3 produces cage $_{3}$-a with sufficient purity. The optimized ROMO reaction conditions were also applicable to four-, six-, and eight-armed 
star-shaped PCLs bearing a norbornene at each chain end (Pre ${ }_{4}$-a, Pre $_{6}$-a, and Pre $_{8}$-a), which afforded the corresponding cage-shaped PCLs with varied arm numbers ( cage $_{4}-\mathbf{a}$, cage $_{\mathbf{6}}-\mathbf{a}$, and cage $_{\mathbf{8}^{-a}}$, respectively) in good yields, typically in the range of 80-97\% (Fig. 3, Tables S3-S5†). Each product was fully characterized by SEC, ${ }^{1} \mathrm{H}$ NMR, and MALDI-TOF MS, which confirmed the successful synthesis of the macromolecular cages (Fig. S9-S18†). It is worth noting that narrowly dispersed macromolecular cages $(\theta=1.06-1.09)$ were obtained without obvious side reactions, despite the increase in the arm-numbers of the precursors. Although some of the obtained macromolecular cages showed high molecular shoulders in their SEC traces, the purity was calculated to be more than $89 \%$, according to the SEC elution peak area. These results suggest that G3-mediated intramolecular ROMO proceeds in preference to the addition of a second G3 to other norbornenyl groups in the same molecule, preventing the formation of possible byproducts such as the tadpole-shaped product. In addition, we have succeeded in controlling the molecular weight of a series of macromolecular cages in an $M_{\mathrm{n}, \mathrm{NMR}}$ range of $\sim 6000-12000$ (Table 1) by simply employing star-shaped PCLs with different molecular weights. Hence, we have established a versatile yet robust synthetic strategy for macromolecular cages based on the intramolecular ROMO that enables the production of a series of macromolecular cages with controlled molecular weights and arm numbers.

Owing to the lack of a universal synthetic strategy, the polymer properties associated with a cage-shaped architecture have never been systematically evaluated, although a comprehensive structural study has been attempted for monocyclic PCLs. ${ }^{34}$ With a series of macromolecular cages with varied arm numbers and molecular weights in hand, we initially investigated the weight-average hydrodynamic diameters $\left(D_{\mathrm{h}}\right)$ and the weight-average intrinsic viscosities $([\eta])$ in THF by employing triple-detection SEC consisting of multiangle light scattering, viscosity, and refractive index detectors (SEC-MALS-Visco). The $D_{\mathrm{h}}$ values of the macromolecular cages are in the range of 3.8-6.8 $\mathrm{nm}$, as summarized in Table 1 and Fig. 4a (see also Fig. S19 $\dagger$ ). The $D_{\mathrm{h}}$ value is dependent on both the arm number and total molecular weight. Fig. $4 \mathrm{~b}$ shows double-logarithmic plots of the $M_{\mathrm{W}, \mathrm{MALS}}$ versus $[\eta]$ for the prepared linear and monocyclic PCLs as well as macromolecular cages, which
Table 1 Molecular characteristics of cyclic polymers and macromolecular cages obtained by intramolecular ROMO

\begin{tabular}{|c|c|c|c|c|c|}
\hline $\begin{array}{l}\text { Macromolecular } \\
\text { cage }\end{array}$ & $M_{\mathrm{n}, \mathrm{SEC}}{ }^{a}$ & $M_{\mathrm{w}, \text { MALS }}{ }^{b}$ & $\Xi^{a}$ & $D_{\mathrm{h}}^{c}(\mathrm{~nm})$ & $\begin{array}{l}\text { Yield } \\
(\%)\end{array}$ \\
\hline ring-a & 7520 & 6280 & 1.09 & 4.6 & 92.3 \\
\hline ring-b & 11700 & 8890 & 1.09 & 5.8 & 92.3 \\
\hline ring-c & 15200 & 11600 & 1.09 & 6.8 & 91.6 \\
\hline cage $_{3}-\mathrm{a}$ & 6770 & 7550 & 1.09 & 4.4 & 92.3 \\
\hline cage $_{3}-b$ & 9360 & 9370 & 1.09 & 5.0 & 80.0 \\
\hline cage $_{3}-c$ & 11500 & 10200 & 1.09 & 5.6 & 84.0 \\
\hline cage $_{4}-\mathbf{a}$ & 6290 & 7420 & 1.08 & 4.2 & 97.0 \\
\hline cage $_{4}-\mathrm{b}$ & 9590 & 9840 & 1.08 & 5.0 & 94.0 \\
\hline cage $_{4}-\mathrm{c}$ & 10700 & 11800 & 1.08 & 5.6 & 91.0 \\
\hline cage $_{6}-a$ & 5120 & 7950 & 1.08 & 3.8 & 91.0 \\
\hline cage $_{6}-b$ & 8570 & 10900 & 1.09 & 5.0 & 98.7 \\
\hline cage $_{6}-\mathrm{c}$ & 10700 & 12800 & 1.07 & 5.2 & 91.2 \\
\hline cage $_{8}-\mathrm{a}$ & 6910 & 8180 & 1.06 & 3.8 & 98.3 \\
\hline cage $_{8}-b$ & 9130 & 10300 & 1.08 & 4.6 & 97.7 \\
\hline cage $_{8}-\mathrm{c}$ & 12000 & 14300 & 1.06 & 5.6 & 84.7 \\
\hline
\end{tabular}

${ }^{a}$ Determined by SEC in THF using PSt standards. ${ }^{b}$ Weight-average absolute molecular weight $\left(M_{\mathrm{w}, \mathrm{MALS}}\right)$ was estimated by SEC-MALSVisco in THF. ${ }^{c}$ Weight-average hydrodynamic diameter $\left(D_{\mathrm{h}}\right)$ was determined through SEC-MALS-Visco measurements in THF by the following equations: $D_{\mathrm{h}}=2 R_{\mathrm{h}}=2\left(3 V_{\mathrm{h}} / 4 \pi\right)^{1 / 3}$ where $V_{\mathrm{h}}$ (hydrodynamic volume) was calculated using the Einstein-Simha equation $\left(V_{\mathrm{h}}=\right.$ $M_{\mathrm{w}, \mathrm{MALS}}[\eta] / 2.5 N_{\mathrm{A}}$, where $N_{\mathrm{A}}$ is Avogadro's number).

clearly indicate a linear relationship between the viscosity and molecular weight. More importantly, the $[\eta]$ values for the macromolecular cages (5.4-14.1 $\mathrm{mg} \mathrm{mL}^{-1}$ ) are apparently lower than those of the corresponding precursors $\left(11.1-22.0 \mathrm{mg} \mathrm{mL}^{-1}\right.$; Tables S2-S5 and Fig. S20†), despite their comparable molecular weights. In addition, the $[\eta]$ values of the macromolecular cages further decrease with increasing arm number. A similar trend was observed in a series of multicyclic polymers. ${ }^{35,36}$ These solution state studies demonstrate that the increase in the arm number or decrease in the molecular weight of the macromolecular cage results in a lower hydrodynamic volume, which supports the possibility of controlling the inner cavity size.

Since PCL is a typical crystalline polymer, its solid state properties are also of particular interest. Thus, the melting temperature $\left(T_{\mathrm{m}}\right)$, crystallinity $\left(X_{\mathrm{WAXD}}\right)$, and lamellar thickness in the PCL crystal long period were then examined by differential scanning calorimetry (DSC), wide-angle X-ray diffraction
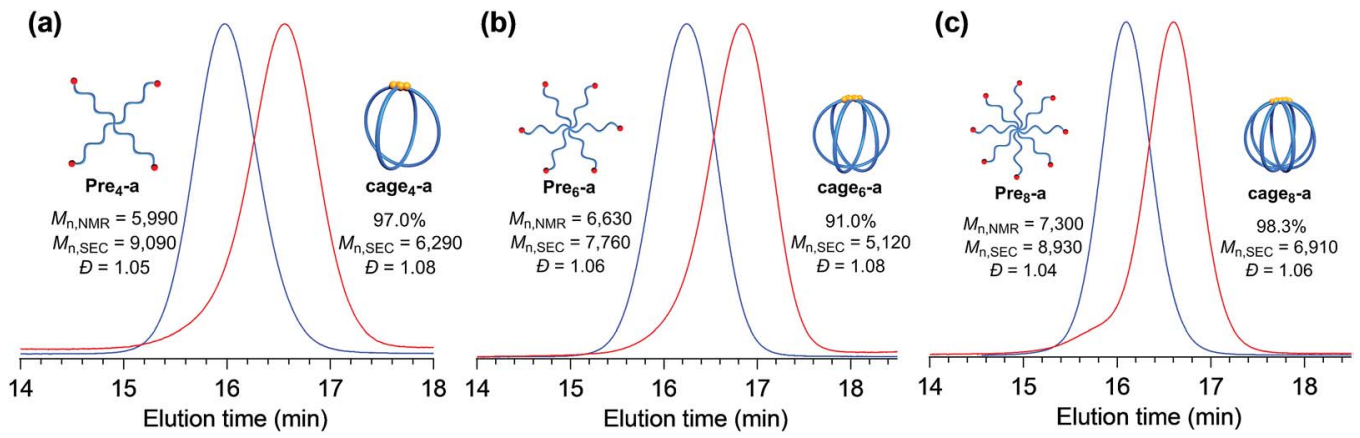

Fig. 3 SEC traces for (a) four-, (b) six-, and (c) eight-armed star-shaped precursors (blue; Pre ${ }_{4}-a$, Pre ${ }_{6}-a$, and Pre $-a$, respectively) and the

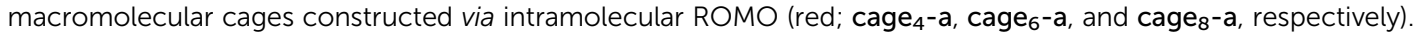


(a) Hydrodynamic diameter $\left(D_{h}\right)$

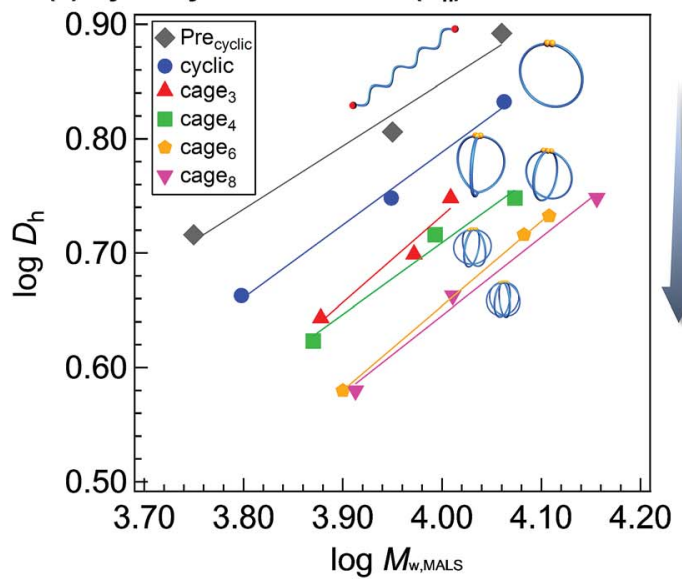

(c) Melting tem perature $\left(T_{m}\right)$ and crystallinity $\left(X_{\text {WAXD }}\right)$

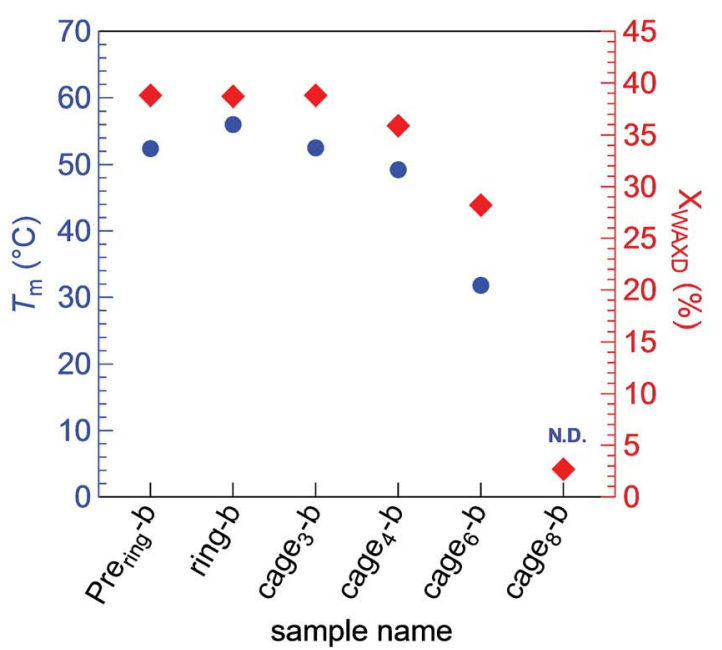

(b) Intrinsic viscosity

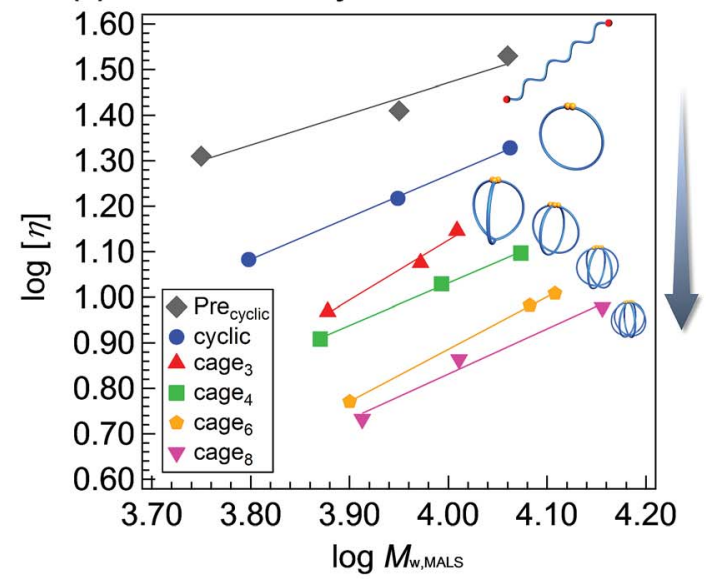

(d) Crystalline lamellar thickness

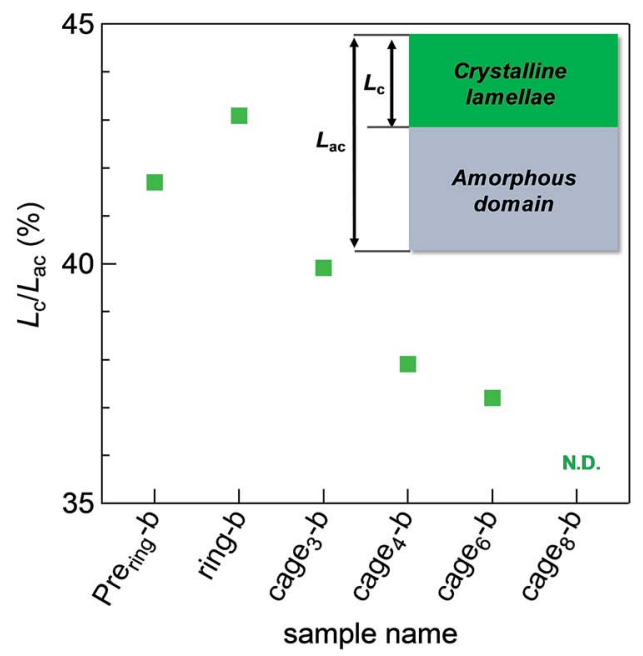

Fig. 4 Structure-property relationships in macromolecular cage systems. (Panels a and b) Double logarithmic plots of $M_{\mathrm{w}, \mathrm{MALS}}$ versus $D_{\mathrm{h}}$ and $[\eta]$

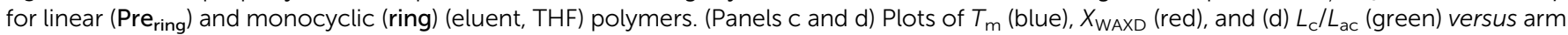
numbers of linear, monocyclic polymers, and macromolecular cages (all the samples had a $M_{n, N M R}$ of $c a$. 9000; from left to right, Pre ring $^{-b}$, ring$b$, cage $3-b$, cage ${ }_{4}-b$, cage $6-b$, and cage ${ }_{8}-b$ ). The inset in panel (d) illustrates the model structure of the long period of the PCL $c r y s t a l$ which consists of a crystalline lamellar and an amorphous domain with thicknesses of $L_{c}$ and $L_{a c}$, respectively. The $T_{m}$ and $L_{c} / L_{a c}$ for cage $-b$ were not determined (N.D.) due to its poor crystallinity.

(WAXD), and small-angle X-ray scattering (SAXS) measurements, respectively. Note that a series of samples discussed in Fig. $4 \mathrm{c}$ and d had a $M_{\mathrm{n}, \mathrm{NMR}}$ of $c a .9000$ and thus their arm lengths are reduced with increasing arm number. The $T_{\mathrm{m}}$ value of the ring-b $\left(56.0^{\circ} \mathrm{C}\right)$ is found to be slightly higher than that of the linear counterpart Pre ring $^{-\mathbf{b}}\left(52.4^{\circ} \mathrm{C}\right)$. Ree and Saalwächter groups also reported a similar trend in the comparison between linear and ring PCLs. ${ }^{34,37}$ In contrast, the $T_{\mathrm{m}}$ and $X_{\mathrm{WAXD}}$ values of macromolecular cages apparently decrease with increasing arm number. For example, the $T_{\mathrm{m}}$ and $X_{\mathrm{WAXD}}$ of cage $\mathbf{6}_{\mathbf{6}}-\mathbf{b}$ are determined to be $31.8{ }^{\circ} \mathrm{C}$ and $28.2 \%$, respectively, which are apparently lower than those of ring-b $\left(56.0{ }^{\circ} \mathrm{C}\right.$ and $38.7 \%$, respectively) and cage $\mathbf{c}_{3}-\mathbf{b}\left(52.5{ }^{\circ} \mathrm{C}\right.$ and $38.8 \%$, respectively). On the other hand, cage $_{\mathbf{8}} \mathbf{- b}$ is found to hardly crystallize. These results implied the distinctive difference in crystallization behaviors between the single cyclic polymer and macromolecular cages. An increase in the arm number causes a decrease in the chain mobility and chain packing ability as well as reduction in each arm length, resulting in less or no crystalline formation in the macromolecular cages (Fig. S23 $\dagger$ ). ${ }^{38,39}$ To further gain an insight into the crystallization behaviors, SAXS analysis was performed on the PCL samples, which provided information about the crystalline lamellar layer formation in crystalline-amorphous two-phase systems (Fig. S24-S28†). Based on the correlation function analysis of the SAXS profiles, ${ }^{40}$ we estimated the ratio of the crystalline lamellae thickness $\left(L_{c}\right)$ and long period ( $L_{\mathrm{ac}}$ ), i.e., $L_{\mathrm{c}} / L_{\mathrm{ac}}$ (Fig. $4 \mathrm{~d}$ ). The $L_{\mathrm{c}} / L_{\mathrm{ac}}$ values of the macromolecular cages decrease with increasing arm number, whereas no significant change is observed in their $L_{\mathrm{ac}}$ (see Tables S1-S5 $\dagger$ ). In a similar manner to the $T_{\mathrm{m}}$ and $X_{\text {WAXD }}$ values, a significant decrease in the lamellae thickness (37.2-43.1\% and N.D.) is observed with increasing arm number, which can also be considered due to the suppressed molecular mobility and diminished chain-packing ability. The increased $L_{\mathrm{c}} / L_{\mathrm{ac}}$ 
value in ring-b, as compared to its linear counterpart, seems to be correlated with its increased $T_{\mathrm{m}}$ value. It is also worth noting that the $T_{\mathrm{m}}, X_{\mathrm{WAXD}}$, and $L_{\mathrm{c}} / L_{\mathrm{ac}}$ of the macromolecular cages tend to be lower than those of the star-shaped precursors, despite their equivalent arm length. This suggests that one additional junction point to construct the cage-shaped architecture can bring about a significant impact on the solid state properties. The difference in the crystallization behaviors between the macromolecular cages and the corresponding star-shaped precursors is more pronounced when the arm number is increased. For example, low molecular weight macromolecular cages having six- and eight-arms (cage $\mathbf{6}_{6}$-a and cage $_{\mathbf{8}}-\mathbf{a} ; M_{\mathrm{n}, \mathrm{NMR}}=$ $c a$. 6000) do not show any evidence of crystallization from the WAXD analysis (Fig. S29-S33†), while their star-shaped precursors displayed distinct scattering peaks corresponding to the PCL crystal structure (Fig. S27, S28, S32 and S33†). Interestingly, cage $_{\mathbf{8}}-\mathbf{b}\left(M_{\mathrm{n}, \mathrm{NMR}}=9530\right)$ with a degree of polymerization of each arm of around eight is still amorphous, despite the fact that the linear caprolactone tetramer can be crystallized. ${ }^{41}$ This demonstrates cage-shaped topological effects on the solid state properties.

\section{Conclusions}

In this study, we have successfully established a robust and versatile synthetic strategy for macromolecular cages with desired arm numbers and sizes based on the intramolecular consecutive ROMO of the highly reactive norbornenyl groups attached to star-shaped polymer precursors. To the best of our knowledge, this study provides the first successful example of constructing multi-ring polymers through chain-reaction-type cyclization. In addition, we were able to systematically evaluate the polymer properties associated with the cage-shaped architecture, which revealed that the hydrodynamic diameter, viscosity, and crystallization behavior of macromolecular cages are strongly affected by the arm number and arm length. The synthetic strategy proposed in this paper should be applicable to a wide range of polymer backbones, enabling access to macromolecular cages with unique structures and functions that will open new avenues for research in supramolecular chemistry and materials science.

\section{Conflicts of interest}

There are no conflicts to declare.

\section{Acknowledgements}

This work was financially supported by MEXT Grant-in-Aid for Challenging Exploratory Research (16K14000), Grant-in-Aid for Scientific Research (B) (16H04152), and Grant-in-Aid for Scientific Research on Innovative Areas "Hybrid Catalysis" (18H04639).

\section{Notes and references}

1 T. R. Cook and P. J. Stang, Chem. Rev., 2015, 115, 7001-7045.
2 D. Zhang, A. Martinez and J. P. Dutasta, Chem. Rev., 2017, 117, 4900-4942.

3 S. Durot, J. Taesch and V. Heitz, Chem. Rev., 2014, 114, 85428578.

4 G. Jutz, P. Van Rijn, B. Santos Miranda and A. Böker, Chem. Rev., 2015, 115, 1653-1701.

5 S. Juul, F. Iacovelli, M. Falconi, S. L. Kragh, B. Christensen, R. Frøhlich, O. Franch, E. L. Kristoffersen, M. Stougaard, K. W. Leong, Y. P. Ho, E. S. Sørensen, V. Birkedal, A. Desideri and B. R. Knudsen, ACS Nano, 2013, 7, 97249734.

6 C. M. Erben, R. P. Goodman and A. J. Turberfield, Angew. Chem., Int. Ed., 2006, 45, 7414-7417.

7 R. McCaffrey, H. Long, Y. Jin, A. Sanders, W. Park and W. Zhang, J. Am. Chem. Soc., 2014, 136, 1782-1785.

8 Y. Ueda, H. Ito, D. Fujita and M. Fujita, J. Am. Chem. Soc., 2017, 139, 6090-6093.

9 M. Yoshizawa, J. K. Klosterman and M. Fujita, Angew. Chem., Int. Ed., 2009, 48, 3418-3438.

10 J. M. Lehn, Pure Appl. Chem., 1978, 50, 871-892.

11 M. Mastalerz, Angew. Chem., Int. Ed., 2010, 49, 5042-5053.

12 T. G. W. Edwardson, K. M. M. Carneiro, C. K. McLaughlin, C. J. Serpell and H. F. Sleiman, Nat. Chem., 2013, 5, 868-875.

13 Y. T. Lai, D. Cascio and T. O. Yeates, Science, 2012, 336, 1129. 14 M. S. Yavuz, Y. Cheng, J. Chen, C. M. Cobley, Q. Zhang, M. Rycenga, J. Xie, C. Kim, K. H. Song, A. G. Schwartz, L. V. Wang and Y. Xia, Nat. Mater., 2009, 8, 935-939.

15 D. Fujita, Y. Ueda, S. Sato, N. Mizuno, T. Kumasaka and M. Fujita, Nature, 2016, 540, 563-566.

16 T. R. Cook, Y. R. Zheng and P. J. Stang, Chem. Rev., 2013, 113, 734-777.

17 B. Zheng, F. Wang, S. Dong and F. Huang, Chem. Soc. Rev., 2012, 41, 1621-1636.

18 G. Polymeropoulos, G. Zapsas, K. Ntetsikas, P. Bilalis, Y. Gnanou and N. Hadjichristidis, Macromolecules, 2017, 50, 13-1290.

19 C. M. Bates and F. S. Bates, Macromolecules, 2017, 50, 3-22. 20 J. E. Poelma, K. Ono, D. Miyajima, T. Aida, K. Satoh and C. J. Hawker, ACS Nano, 2012, 6, 10845-10854.

21 J. Jeong, K. Kim, R. Lee, S. Lee, H. Kim, H. Jung, M. A. Kadir, Y. Jang, H. B. Jeon, K. Matyjaszewski, T. Chang and H. J. Paik, Macromolecules, 2014, 47, 3791-3796.

22 Y. Tezuka, A. Tsuchitani, Y. Yoshioka and H. Oike, Macromolecules, 2003, 36, 65-70.

23 Y. Tezuka and K. Fujiyama, J. Am. Chem. Soc., 2005, 127, 6266-6270.

24 Y. Satoh, H. Matsuno, T. Yamamoto, K. Tajima, T. Isono and T. Satoh, Macromolecules, 2017, 50, 97-106.

25 S. C. Radzinski, J. C. Foster, R. C. Chapleski, D. Troya and J. B. Matson, J. Am. Chem. Soc., 2016, 138, 6998-7004.

26 Y. Xia and R. H. Grubbs, Macromolecules, 2009, 50, 197-198. 27 A. Li, Z. Li, S. Zhang, G. Sun, D. M. Policarpio and K. L. Wooley, ACS Macro Lett., 2012, 1, 241-245.

28 K. Makiguchi, T. Satoh and T. Kakuchi, Macromolecules, 2011, 44, 1999-2005.

29 B. A. Laurent and S. M. Grayson, Chem. Soc. Rev., 2009, 38, 2202-2213. 
30 T. Isono, T. Sasamori, K. Honda, Y. Mato, T. Yamamoto, K. Tajima and T. Satoh, Macromolecules, 2018, 51, 3855-3864.

31 M. D. Hossain, D. Valade, Z. Jia and M. J. Monteiro, Polym. Chem., 2012, 3, 2986.

32 H. Misaka, R. Kakuchi, C. Zhang, R. Sakai, T. Satoh and T. Kakuchi, Macromolecules, 2009, 42, 5091-5096.

33 J. N. Hoskins and S. M. Grayson, Macromolecules, 2009, 42, 6406-6413.

34 L. Xiang, W. Ryu, H. Kim and M. Ree, Polymers, 2018, 10, 577. 35 M. D. Hossain, J. C. Reid, D. Lu, Z. Jia, D. J. Searles and M. J. Monteiro, Biomacromolecules, 2018, 19, 616-625.
36 A. Pipertzis, M. D. Hossain, M. J. Monteiro and G. Floudas, Macromolecules, 2018, 51, 1488-1497.

37 K. Schäler, E. Ostas, K. Schröter, T. Thurn-Albrecht, W. H. Binder and K. Saalwächter, Macromolecules, 2011, 44, 2743-2754.

38 J. L. Wang and C. M. Dong, Polymer, 2006, 47, 3218-3228.

39 Y. Tezuka, T. Ohtsuka, K. Adachi, R. Komiya, N. Ohno and N. Okui, Macromol. Rapid Commun., 2008, 29, 1237-1241.

40 B. Goderis, H. Reynaers, M. H. J. Koch and V. B. F. Mathot, J. Polym. Sci., Part B: Polym. Phys., 1999, 1, 1715-1738.

41 K. Takizawa, C. Tang and C. J. Hawker, J. Am. Chem. Soc., 2008, 130, 1718-1726. 